\title{
Clotting Factors and Eicosanoids Protect against Nematode Infections
}

\author{
Pavel Hyrsl ${ }^{a, b}$ Pavel Dobes ${ }^{a, b}$ Zhi Wang ${ }^{a}$ Thomas Hauling ${ }^{a}$ \\ Christine Wilhelmsson $^{a} \quad$ Ulrich Theopold ${ }^{a}$ \\ ${ }^{a}$ Department of Molecular Biology and Functional Genomics, Stockholm University, Stockholm, Sweden; \\ ${ }^{b}$ Department of Animal Physiology and Immunology, Institute of Experimental Biology, Masaryk University, \\ Brno, Czech Republic
}

\begin{abstract}
Key Words
Drosophila melanogaster $\cdot$ Eicosanoids $\cdot$ Entomopathogenic nematodes $\cdot$ Heterorhabditis bacteriophora $\cdot$ Coagulation •

Transglutaminase $\cdot$ Photorhabdus luminescens
\end{abstract}

\begin{abstract}
We show that hemolymph clotting protects Drosophila melanogaster against infections with an entomopathogenic nematode and its symbiotic bacterium. We also provide biochemical and genetic evidence for an involvement of eicosanoids in the same infection model. Taken together, our results confirm the conserved nature of the immune function of clot formation.

Copyright $\odot 2010$ S. Karger AG, Basel
\end{abstract}

\section{Introduction}

Insect hemolymph clotting serves to seal wounds and has been proposed to contribute to preventing migration of microbes past the wound site [1], similar to other invertebrates [2]. Using proteomics and bioinformatics, we have previously identified a number of candidate clotting factors and studied their function upon wounding and in infection models [3-5]. The enzyme transglutaminase (TG) and its vertebrate homologue factor XIIIa are amongst the best-conserved clotting factors and help to sequester bacteria at the wound site in both insects and humans. Recent evidence for an immune function of TG derives from an infection model that involves insect pathogenic nematodes and their symbiotic bacteria [6]. Infection with nematodes such as Heterorhabditis bacteriophora involves the release of symbiotic bacteria (Photorhabdus luminescens) from the gut, which helps the nematode to suppress host immunity and eventually kill the host [7]. Genetic studies in Drosophila show that despite the fact that antimicrobial peptides are induced in Heterorhabditis/Photorhabdus-infected hosts, both major pathways of immune induction (Toll and imd) appeared dispensable for the response against the nematodes and their bacteria [8]. We have previously shown that TG plays a role in the defense against Heterorhabditis/Photorhabdus; Drosophila larvae with reduced TG levels were more sensitive to nematode infections [6]. Upon contact with Photorhabdus, hemolymph TG targets microbial surfaces, leading to the deposition of small aggregates (microclots). Here, we screened clotting factors other than TG for their effects during a Heterorhabditis/Photorhabdus infection. We provide further evidence for an immune function of the clot and show that eicosanoids, which are required during blood clotting [9], also regulate the response against nematodes and their bacteria.

\section{KARGER}

Fax +41613061234

E-Mail karger@karger.ch

www.karger.com
(C) 2010 S. Karger AG, Basel

$1662-811 X / 11 / 0031-0065 \$ 26.00 / 0$

Accessible online at:

www.karger.com/jin
Dr. Ulrich Theopold

Department of Molecular Biology and Functional Genomics

University of Stockholm

SE-10691 Stockholm (Sweden)

Tel. +46816 4181, Fax +46816 6488, E-Mail uli@molbio.su.se 


\section{Materials and Methods}

\section{Fly Strains}

RNAi lines from the Vienna collection [10] were used. To account for differences in knock-down due to position effects, we used several lines for a given gene. These included lines for Tiggrin (stocks 28255 and 100036), gp150 (stocks 899, 900 and 100134), hemomucin (stocks 1278, 1279 and 100286), Fbp1 (stock 37881) and CG14507 (stocks 106109 and 35713). For gp150 lines, we determined the level of knock-down by measuring the strength of the peanut agglutinin signal that detects gp150 [11], normalized to an internal Coomassie-stained control (hexamerin) in hemolymph samples from the different lines. With this method, we found stock 100134 to work best (50\% knock-down; a previously published line [11] did not show efficient knock-down with the hemese driver). The line 100134 showed a significant increase in mortality after $48 \mathrm{~h}(\mathrm{p}=0.0052$, as shown in figure 1 ; note that a second line from the Vienna collection also showed increased mortality at $\mathrm{p}=0.027$, data not shown). The RNAi line targeting Fondue and its level of knock-down had been characterized before [12]. The RNAi line for NimC1 was a kind gift from I. Ando, Szeged, Hungary. Gal4-expressing driver lines with specificity for either the fat body (pplGal4), hemocytes (HeGal4) or ubiquitously expressed Gal4 (ActinGal4) were used. $W^{1118}$ wild-type and $B c$ mutant flies were obtained from the Bloomington fly stock center (Bloomington, Ind., USA), and $i m d^{1}$ mutant flies were a kind gift from B. Lemaitre. Note that $i m d^{1}$ is a weak hypomorphic allele, a fact that might contribute to the lack of a phenotype upon nematode infection.

\section{Identification of TG Substrates}

TG substrates were identified as described before [5] using mass spectrometry.

\section{Clot Preparation}

Clots were prepared from larvae expressing a Fondue-GFP fusion protein [13] using the hanging drop method [14].

\section{Identification of Microclots}

The eicosanoid biosynthesis inhibitor dexamethasone was injected into wild-type larvae $\left(W^{1118}\right)$ at a concentration of $5 \mu \mathrm{g} / \mu \mathrm{l}$ in PBS. Bacteria were injected after $12 \mathrm{~h}$. One hour later, hemolymph was retrieved from the larvae to perform a B-cad incorporation assay as described elsewhere [6]. As controls, larvae injected with PBS were used.

\section{Infection Experiments}

Nematode infection of larvae was performed as described previously [6], using H. bacteriophora (strain H222) naturally carrying $P$. luminescens ssp. kayaii. All experiments were run in triplicate using 48 larvae per group at different temperatures and nematode doses $\left(22^{\circ} \mathrm{C}\right.$ and 100 nematodes/larva for inhibitor injections, $25^{\circ} \mathrm{C}$ and 100 nematodes/larva for the experiments shown in figure $3 \mathrm{C}$ or $29^{\circ} \mathrm{C}$ and 25 nematodes/larva for the experiments shown in figure 1 and $3 \mathrm{~B}$ ).

\section{Eicosanoid Inhibitor Injections}

The eicosanoid biosynthesis inhibitors esculetin (6,7-dihydroxycoumarin), dexamethasone $[(11 \beta, 16 \alpha)$-9-fluoro-11-17,21trihydroxy-16-methylpregna-1,4-diene-3, 20-dione] and indo-
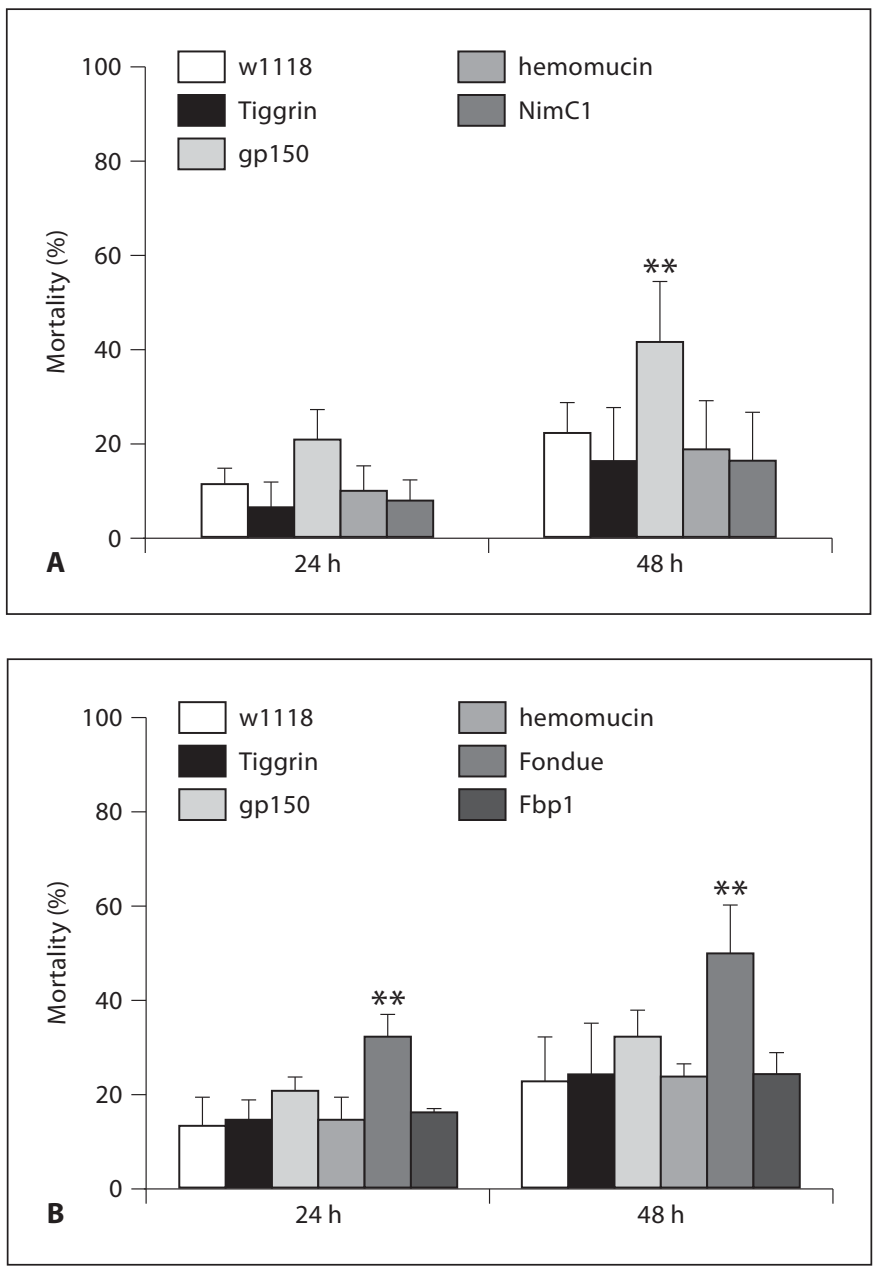

Fig. 1. Identification of clot components that contribute to the defense against Heterorhabditis/Photorhabdus. RNAi lines for individual clotting factors were crossed either with a hemocyte-specific (A) or a fat body-specific driver (B) and infected with Heterorhabditis/Photorhabdus, and mortality was scored after 24 and $48 \mathrm{~h}$ (see Materials and Methods for further details on infection and efficiency of the knock-down). Data represent means \pm SD. ** $\mathrm{p}<0.01$.

methacin [1-(4-chlorobenzoyl)-5-methoxy-2-methyl-3-indoleacetic acid], all from Sigma-Aldrich (St. Louis, Mo., USA), were used. Seventy-two-hour-old larvae of $D$. melanogaster were injected with $0.05 \mu \mathrm{l}$ of inhibitors dissolved in PBS $(5 \mu \mathrm{g} / \mu \mathrm{l}) \mathrm{using}$ the automatic nanoliter injector Nanoject II (Drummond Scientific, Pa., USA). As controls, untreated larvae and larvae injected with the same volume of PBS were used. The larvae were exposed to nematode infection $12 \mathrm{~h}$ after injection. Data were analyzed using Student's t test and comparison with the PBS-injected control.
66

J Innate Immun 2011;3:65-70
Hyrsl/Dobes/Wang/Hauling/

Wilhelmsson/Theopold 

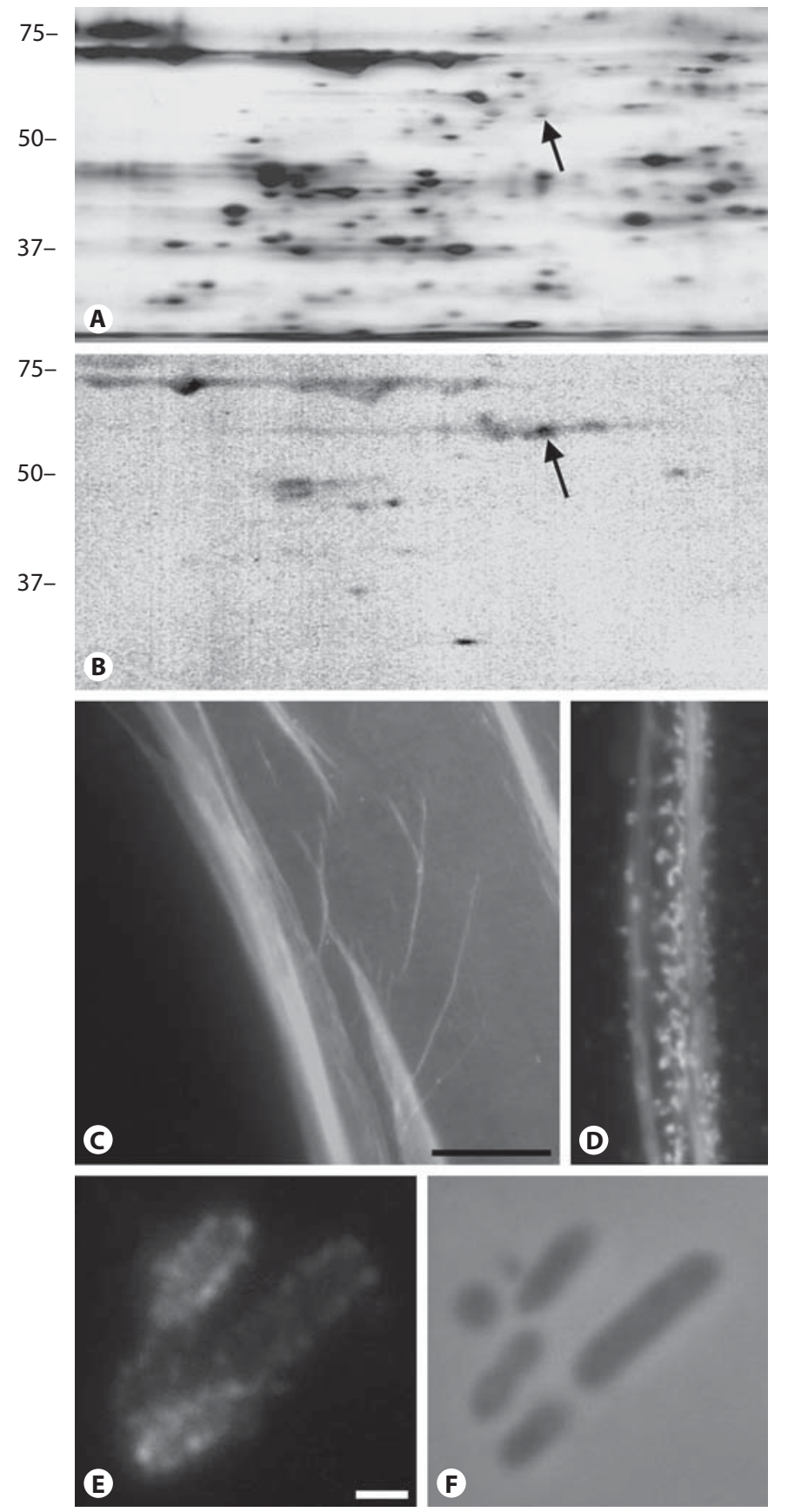

Fig. 2. Fondue contributes to both the clot matrix and microclots on Photorhabdus. A, B Hemolymph was incubated with a biotin-containing substrate (biotin-cadaverine) which labels TG substrates. The sample was split into two parts, which were run in parallel and processed either by staining with Coomassie $(\mathbf{A})$ or blotting and probing with biotin-specific streptavidin (B). The arrows indicate the position of Fondue, which is among the preferred TG substrates. The $\mathrm{pH}$ range was between 3 and 10 . C, D Fondue is part of the clot matrix and microclots. A clot was prepared as described from larvae expressing a Fondue-GFP fusion protein [13] and visualized using fluorescence microscopy. In C, a part of the clot matrix, which has partially folded back onto

\section{Results}

To test whether clotting factors other than TG are required for the response against nematodes and their bacteria we infected knock-down (RNAi) lines for previously identified clotting factors including hemomucin [15], gp150 (or Eig71Ee, CG7604 [11]), Fbpl, Tiggrin and Fondue $[4,5]$. To minimize side effects due to ubiquitous knock-down, we relied on known information on the expression of these factors using driver lines with specificity for the fat body, hemocytes or both. Significant increases in susceptibility were observed when gp150 expression was reduced in hemocytes (fig. 1A). Similarly, knock-down of Fondue in the fat body (fig. 1B) increased mortality after nematode infection. In contrast, knockdown of the phagocytic receptor Nimrod C1 in hemocytes had no effect, adding further support to the idea that phagocytosis does not play a major role (fig. 1A) [6]. Nor did knock-down of any other clotting factor show any effects (fig. 1). Although due to the partial nature of knock-downs an influence of genes that did not show an effect on mortality cannot be excluded, these results further support a role for clotting factors in the response against nematodes and their bacteria.

Fondue is one of the preferred hemolymph substrates for TG (fig. 2A, B) [5]. To get further insight into its function, we decided to trace Fondue using a fusion construct with GFP [13]. In hemolymph clots, the protein is part of both the fibrous matrix that forms the clot itself and local aggregates that resemble microclots (fig. 2C, D). When Photorhabdus was injected into larvae expressing the fusion protein, microclots were also found on the surface of the bacteria (fig. 2E, F). Taken together, these results confirm the importance of clot formation as part of the response against entomopathogenic nematodes.

itself (left part), is shown, while in $\mathbf{D}$ the matrix has folded up more extensively into longer strands, part of which is shown. E, F Microclots bind to bacteria. Photorhabdus were injected into Fondue-GFP-expressing larvae and retrieved by bleeding followed by immunohistology with a GFP-specific and a TRITC-conjugated secondary antibody. Note the punctate structures on the bacteria (microclots), which had been observed before using TG-specific reagents [6] (fluorescence and phase contrast exposure of the same field are shown). Scale bars $=10 \mu \mathrm{m}$ (C, D, same magnification) and $1 \mu \mathrm{m}(\mathbf{E})$. 


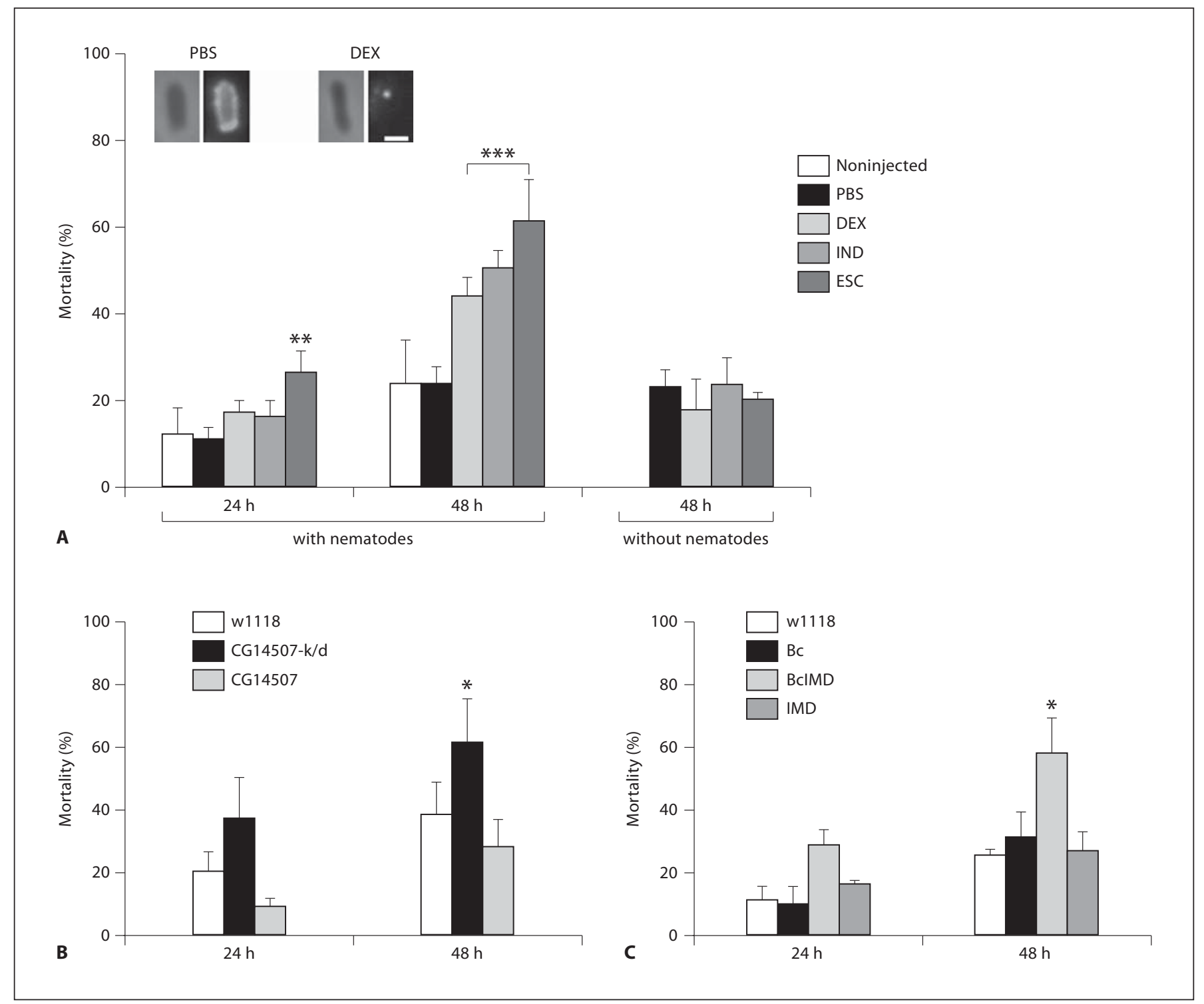

Fig. 3. Eicosanoids and the imd pathway contribute to immunity against Heterorhabditis/Photorhabdus. A Infection experiments were performed after previous injection of eicosanoid biosynthesis inhibitors [dexamethasone (DEX), indomethacine (IND) and esculetin (ESC)]. Control larvae were injected with the inhibitors alone without subsequent infection with nematodes, and survival was scored after $48 \mathrm{~h}$. The inset shows bacteria introduced into dexamethasone- and PBS-injected larvae, which were retrieved after $1 \mathrm{~h}$ and treated as described elsewhere [6] to detect micro-

Since eicosanoids are well-established regulators of blood clotting [9] and have been implicated in insect immunity $[16,17]$, we speculated that they might also play a role during Heterorhabditis/Photorhabdus infection. To test this, we injected wild-type larvae with known in-

clots. Scale bar $=1 \mu \mathrm{m}$. Mortality was scored after the indicated times. B Mortality after infection of knock-down (k/d; using the actin driver line) of the lines for secreted PLA 2 (CG14507), a control cross (w1118, crossed with the actin driver line) and the responder line alone. C Infection of control larvae, $i m d^{1}$ and $B c$ mutants alone and $i m d^{1} / B c$ double mutants reveals an influence of the imd pathway in the absence of melanization. ${ }^{*} \mathrm{p}<0.05$; ${ }^{* *} \mathrm{p}<0.01 ;{ }^{* * *} \mathrm{p}<0.001$. All inhibitors showed the same significance level at $48 \mathrm{~h}$ in $\mathbf{A}$.

hibitors of eicosanoid synthesis prior to infection with nematodes (fig. 3A). All three inhibitors led to an increase in mortality $48 \mathrm{~h}$ after infection, which suggests that eicosanoids or related lipids do play a role and agrees with the observation that Photorhabdus induces immu- 
nosuppression in its host by inhibiting phospholipase A2 $\left(\mathrm{PLA}_{2}\right)$, the most upstream enzyme in the eicosanoid biosynthesis pathway [18]. In addition, the formation of microclots was inhibited in the presence of dexamethasone (fig. 3A, inset). To obtain independent genetic evidence for a role of eicosanoids in this infection model, we performed similarity searches for Drosophila homologues of PLA $\mathrm{P}_{2}$. Amongst the phospholipases identified, we focused on the secretory PLA 2 CG14507, since secretory phospholipases had been implicated in the response against entomopathogenic bacteria [19] and CG14507 had previously been shown to be induced upon wounding by laser ablation [20]. CG14507 RNAi larvae were indeed more susceptible to nematodes and their bacteria (fig. 3B), providing additional genetic evidence for a function of eicosanoids in insect immunity. Conversely, knock-down of two genes which had been electronically annotated as potentially involved in eicosanoid synthesis (CG10602 and CG1742, Flybase) had no effect (not shown). Since eicosanoid synthesis had previously been shown to act in parallel to the imd pathway, and recent evidence suggests a complex role for the imd pathway in the response against Photorhabdus [21, 22], we also decided to reevaluate the role of IMD in the response against Heterorhabditis/Photorhabdus (fig. 3C). To facilitate detection of $i m d$-dependent effects, we performed the infection in $B c$ mutants, which lack phenoloxidase activity due to a defect in hemocyte development [23], and in $B c /$ imd double mutants. The double mutants were indeed more sensitive, and since $B c$ mutants alone showed no increased susceptibility, this result further supports the idea that eicosanoid metabolism is at least partially connected to imd signaling [21] and is in line with previous results which show synergy between $B c$ and $i m d^{1}$ [24].

\section{Discussion}

Here we confirm and expand our previous findings and show that hemolymph clotting is an integral part of immunity. We also observe that both steroidal and nonsteroidal inhibitors of eicosanoid biosynthesis interfere with nematode infections, findings which are in line with previous work in other insect hosts and provide, to our knowledge, the first direct genetic evidence for a role of eicosanoids in insect immunity. This supports earlier biochemical data $[16,18,25]$ and opens up the interesting perspective that regulation of clotting through eicosanoids has been phylogenetically conserved. Future work will reveal the exact nature of the lipids produced in this pathway in insects. It should be noted that similar to other invertebrates that produce eicosanoids, Drosophila lacks clearly identifiable homologues of many key enzymes in their synthesis [9]. These include human pathogens such as Leishmania and trypanosomes where synthesis relies on distant members of the vertebrate enzymes or on proteins that have acquired similar activities independently [26]. The work presented here shows that genetic screens may open up new pathways to identify invertebrate enzymes that produce lipid regulators of innate immunity.

\section{Acknowledgements}

We thank Carina Krützmann and Kathrin Handge for help with this project, Fredrik Tholander for useful discussions and the anonymous reviewers for their helpful suggestions. Our research is supported by research grants from the Swedish Research Council (U.T.), the Carl-Tryggers Foundation (U.T. and P.H.) and the Grant Agency of the Czech Republic (P.H.; GA206/09/P470).

\section{References}

1 Theopold U, Dushay MS: Mechanisms of Drosophila immunity - an innate immune system at work. Curr Immunol Rev 2007;3: 276-288.

2 Kawabata S, Koshiba T, Shibata T: The lipopolysaccaride-activated innate immune response network of the horseshoe crab. Invertebr Surv J 2009;6:59-77.

$\checkmark 3$ Theopold U, Li D, Fabbri M, Scherfer C, Schmidt O: The coagulation of insect hemolymph. Cell Mol Life Sci 2002;59:363-372.
-4 Scherfer C, Karlsson C, Loseva O, et al: Isolation and characterization of hemolymph clotting factors in Drosophila melanogaster by a pullout method. Curr Biol 2004;14:625629.

5 Karlsson C, Korayem AM, Scherfer C, Loseva O, Dushay MS, Theopold U: Proteomic analysis of the Drosophila larval hemolymph clot. J Biol Chem 2004;279:52033-52041.

-6 Wang Z, Wilhelmsson C, Hyrsl P, et al: Pathogen entrapment by transglutaminase a conserved early innate immune mechanism. PLoS Pathog 2010;6:e1000763.
7 Ffrench-Constant R, Waterfield N, Daborn P, et al: Photorhabdus: towards a functional genomic analysis of a symbiont and pathogen. FEMS Microbiol Rev 2003;26:433-456.

$>8$ Hallem EA, Rengarajan M, Ciche TA, Sternberg PW: Nematodes, bacteria, and flies: a tripartite model for nematode parasitism. Curr Biol 2007;17:898-904.

9 Simmons DL, Botting RM, Hla T: Cyclooxygenase isozymes: the biology of prostaglandin synthesis and inhibition. Pharmacol Rev 2004;56:387-437. 
10 Dietzl G, Chen D, Schnorrer F, et al: A genome-wide transgenic RNAi library for conditional gene inactivation in Drosophila. Nature 2007;448:151-156.

-11 Korayem AM, Fabbri M, Takahashi K, et al: A Drosophila salivary gland mucin is also expressed in immune tissues: evidence for a function in coagulation and the entrapment of bacteria. Insect Biochem Mol Biol 2004; 34:1297-1304.

12 Scherfer C, Qazi MR, Takahashi K, et al: The Toll immune-regulated Drosophila protein Fondue is involved in hemolymph clotting and puparium formation. Dev Biol 2006;295: 156-163.

13 Lindgren M, Riazi R, Lesch C, Wilhelmsson C, Theopold U, Dushay MS: Fondue and transglutaminase in the Drosophila larval clot. J Insect Physiol 2008;54:586-592.

$\checkmark 14$ Bidla G, Lindgren M, Theopold U, Dushay MS: Hemolymph coagulation and phenoloxidase in Drosophila larvae. Dev Comp Immunol 2005;29:669-679.

15 Theopold U, Schmidt O: Helix pomatia lectin and annexin $\mathrm{V}$, two molecular probes for insect microparticles: possible involvement in hemolymph coagulation. J Insect Physiol 1997;43:667-674
16 Stanley D, Miller J, Tunaz H: Eicosanoid actions in insect immunity. J Innate Immun 2009;1:282-290.

17 Shrestha S, Park Y, Stanley D, Kim Y: Genes encoding phospholipases A2 mediate insect nodulation reactions to bacterial challenge. J Insect Physiol 2010;56:324-332.

18 Kim Y, Ji D, Cho S, Park Y: Two groups of entomopathogenic bacteria, Photorhabdus and Xenorhabdus, share an inhibitory action against phospholipase A2 to induce host immunodepression. J Invertebr Pathol 2005;89: 258-264.

19 Shrestha S, Kim Y: Biochemical characteristics of immune-associated phospholipase $\mathrm{A}(2)$ and its inhibition by an entomopathogenic bacterium, Xenorhabdus nematophila. J Microbiol 2009;47:774-782.

20 Stramer B, Winfield M, Shaw T, Millard TH, Woolner S, Martin P: Gene induction following wounding of wild-type versus macrophage-deficient Drosophila embryos. EMBO Rep 2008;9:465-471.

21 Yajima M, Takada M, Takahashi N, et al: A newly established in vitro culture using transgenic Drosophila reveals functional coupling between the phospholipase A2generated fatty acid cascade and lipopolysaccharide-dependent activation of the immune deficiency (imd) pathway in insect immunity. Biochem J 2003;371:205-210.
22 Aymeric JL, Givaudan A, Duvic B: Imd pathway is involved in the interaction of Drosophila melanogaster with the entomopathogenic bacteria, Xenorhabdus nematophila and Photorhabdus luminescens. Mol Immunol 2010;47:2342-2348.

23 Rizki RM, Rizki TM: Encapsulation of parasitoid eggs in phenoloxidase-deficient mutants of Drosophila melanogaster. J Insect Physiol 1990;36:523-529.

24 Braun A, Hoffmann JA, Meister M: Analysis of the Drosophila host defense in domino mutant larvae, which are devoid of hemocytes. Proc Natl Acad Sci USA 1998;95: 14337-14342.

25 Park Y, Kim Y: Xenorhabdus nematophilus inhibits p-bromophenacyl bromide (BPB)sensitive PLA2 of Spodoptera exigua. Arch Insect Biochem Physiol 2003;54:134-142.

26 Kubata BK, Duszenko M, Martin KS, Urade Y: Molecular basis for prostaglandin production in hosts and parasites. Trends Parasitol 2007;23:325-331. 